\title{
A summary of the Transfusion Error Surveillance System: 2008 - 2011
}

\author{
Mounchili $A^{1 *}$, Leduc $S^{1}$, Archibald $C^{1}$, Miller $\mathrm{J}^{1}$, Hyson $\mathrm{C}^{1}$ \\ ${ }^{1}$ Centre for Communicable Diseases and Infection Control, Public Health Agency of Canada, Ottawa, ON \\ ${ }^{*}$ Correspondence: aboubakar.mounchili@phac-aspc.gc.ca
}

\section{Abstract}

Background: Although Canada has one of the safest blood systems in the world, transfusion errors can occur at any time from the moment of collection through to the transfusion of blood and blood products. The Transfusion Error Surveillance System (TESS) was initiated by the Public Health Agency of Canada (the Agency) to monitor transfusion-related errors occurring at any point in the transfusion chain.

Objective: To offer an analysis of the TESS data reported from 2008 to 2011.

Methods: Between 2008 and 2011, 12 to 15 hospitals from four provinces participated in the TESS. Reports on all transfusion-related errors were sent electronically on a quarterly basis to the Agency where they were consolidated, cleaned, validated and analyzed. Different types of transfusion errors were categorized by time of discovery (pre- or post-transfusion) and their potential impact on the patient's health. The occurrence rates of different types of errors were calculated using corresponding denominator data. Results were grouped by the transfusion capacity of reporting hospitals.

Results: Between 2008 and 2011, a total of 34,088 transfusion-related errors were reported. Of these, 33,622 $(98.6 \%)$ were detected prior to transfusion. The most commonly reported were errors related to the collection $(40.1 \%)$ and handling $(10.4 \%)$ of blood samples. Of the remaining $466(1.4 \%)$ that were detected after transfusion, 66 were of high potential severity and 16 of them resulted in adverse reactions in recipients. Inappropriate / incorrect / no product order accounted for over $56 \%(n=9)$ of these errors and the most common adverse reaction was transfusion-associated circulatory overload which occurred in eight $(50 \%)$ of the patients that developed adverse reactions.

Conclusion: The TESS data from 2008 to 2011 demonstrates that blood transfusions are both safe and efficient in Canadian hospitals participating in the surveillance and also highlights the most common and most harmful errors that may be targeted for corrective actions.

\section{Introduction}

Blood transfusions are one of the key components of Canada's healthcare system. Each year in Canada, blood transfusions are used to treat people suffering from blood loss, anemia and cancer. Errors that can occur along the blood transfusion chain either before or after the actual transfusion may result in blood type (ABO) incompatibilities, administrative delays, product wastage and even inappropriate transfusions. These errors have the potential to impact patient safety and increase costs incurred by the healthcare system. In 2005, in recognition of the importance of transfusion safety in Canada, the Public Health Agency of Canada (the Agency) developed the Transfusion Error Surveillance System (TESS) to monitor unexpected, unplanned deviations from standard operating procedures or applicable laws and regulations, usually attributable to a human or system problem that could adversely affect the safety, efficacy or quality of blood, blood products and/or the safety of recipients. Outputs of the TESS are valuable at more than one level as they not only identify where and when most high potential severity errors occur in the transfusion chain, they also provide a benchmark for national and international comparisons. Most importantly, they assist in the development and assessment of transfusion safety measures. This is a summary of the $2008-2011$ TESS Report (1). 


\section{Methods}

Between 2008 and 2011, data on various types of transfusion-related errors were reported by a number of hospitals from four Canadian provinces / territories: 12 in 2008, 14 in 2009 and 15 in 2010 and 2011. Transfusion errors were detected within participating hospitals using various methods such as systematic quality control (chart audit, record review, real-time prospective transfusion audit), scheduled quality assurance control, supervisory reports and reporting by any other individual. However, due to the lack of standardization and systematic implementation across participating hospitals, errors detected through scheduled quality control or supervisory reports were excluded from the analysis.

The reporting process began with the individuals who discovered the event, whether or not they were involved. Following detection of a transfusion-related error at a hospital participating in the TESS, non-nominal data about the error and the patient involved were collected by the hospital using an online reporting form. The point in the transfusion chain at which the error occurred was also recorded together with the point at which the error was detected. Given the level of details recorded, a classification system was used to differentiate reported errors by types and sub-types and, to ensure high consistency in the classification across provinces and territories participating in the TESS, the Agency organized regular monthly error coding exercises where the provincial and territorial blood coordinating offices' staff submitted and discussed complex cases encountered in hospitals within their respective jurisdictions.

Data received from the provincial and territorial blood coordinating offices was consolidated into one Excel file, reviewed, validated and exported into the statistical package, Stata, for further statistical analysis. The occurrence rate of each type of error was calculated separately by dividing the total number of errors by its corresponding denominator and converting the result into 100,000 units of products received, requested, prepared, issued or per 100,000 samples received depending on the error type. Also, dividing the denominator (total number of units of products requested / received / issued / prepared or samples received) by the number of reported errors allowed the computation of the risk of a single case of individual type of errors.

Results were categorized by the size of hospitals which was determined based on the volume of blood transfusions performed annually. Hospitals that transfused less than 2,000 units of products were considered small capacity hospitals; whereas those transfusing between 2,000 and 10,000 or more were considered medium or large capacity hospitals.

\section{Results}

From 2008 to 2011, 34,088 transfusion errors that met the surveillance criteria were captured by the TESS (Table 1). The total annual errors reported ranged from a minimum of 8,253 to a maximum of 8,917 recorded respectively in 2009 and 2010 . Only about $7.1 \%(n=2,430)$ were from hospitals of small transfusion capacity compared to $19.4 \%(n=6,617)$ and $73.5 \%(25,041)$ respectively for hospitals of medium and large capacity.

Table 1: Transfusion errors reported by hospitals of various transfusion capacities, TESS $2008-2011$.

\begin{tabular}{|c|c|c|c|c|c|c|c|c|}
\hline \multirow[t]{2}{*}{ Type of Transfusion error } & \multicolumn{2}{|c|}{$\begin{array}{c}\text { Small } \\
\text { (less than 2,000 } \\
\text { transfusions per year) }\end{array}$} & \multicolumn{2}{|c|}{$\begin{array}{c}\text { Medium } \\
(2,000 \text { to } 10,000 \\
\text { transfusions per year })\end{array}$} & \multicolumn{2}{|c|}{$\begin{array}{c}\text { Large } \\
\text { (more than } 10,000 \\
\text { transfusions per year) }\end{array}$} & \multicolumn{2}{|c|}{ Overall } \\
\hline & Freq. & $\%$ & Freq. & $\%$ & Freq. & \begin{tabular}{l|l}
$\%$ & -1
\end{tabular} & Freq. & $\%$ \\
\hline Distributor codes & 42 & $1.7 \%$ & 631 & $9.5 \%$ & 1,069 & $4.3 \%$ & 1,742 & $5.1 \%$ \\
\hline Inventory management & 14 & $0.6 \%$ & 52 & $0.8 \%$ & 94 & $0.4 \%$ & 160 & $0.5 \%$ \\
\hline Product check-in & 62 & $2.6 \%$ & 390 & $5.9 \%$ & 1,030 & $4.1 \%$ & 1,482 & $4.3 \%$ \\
\hline Product request & 32 & $1.3 \%$ & 652 & $9.9 \%$ & 1,989 & $7.9 \%$ & 2,673 & $7.8 \%$ \\
\hline Product selection & 7 & $0.3 \%$ & 32 & $0.5 \%$ & 74 & $0.3 \%$ & 113 & $0.3 \%$ \\
\hline
\end{tabular}




\begin{tabular}{|l|r|r|r|r|r|r|r|r|}
\hline Request for pick-up & 32 & $1.3 \%$ & 254 & $3.8 \%$ & 628 & $2.5 \%$ & 914 & $2.7 \%$ \\
\hline Sample collection & 225 & $9.3 \%$ & 1,338 & $20.2 \%$ & 12,117 & $48.4 \%$ & 13,680 & $40.1 \%$ \\
\hline Sample handling & 331 & $13.6 \%$ & 606 & $9.2 \%$ & 2,610 & $10.4 \%$ & 3,547 & $10.4 \%$ \\
\hline Sample receipt & 945 & $38.9 \%$ & 137 & $2.1 \%$ & 1,042 & $4.2 \%$ & 2,124 & $6.2 \%$ \\
\hline Sample testing & 587 & $24.2 \%$ & 481 & $7.3 \%$ & 1,408 & $5.6 \%$ & 2,476 & $7.3 \%$ \\
\hline Unit issue & 29 & $1.2 \%$ & 167 & $2.5 \%$ & 249 & $1.0 \%$ & 445 & $1.3 \%$ \\
\hline Unit manipulation & 53 & $2.2 \%$ & 129 & $1.9 \%$ & 340 & $1.4 \%$ & 522 & $1.5 \%$ \\
\hline Unit storage & 3 & $0.1 \%$ & 16 & $0.2 \%$ & 51 & $0.2 \%$ & 70 & $0.2 \%$ \\
\hline Unit transfusion & 40 & $1.6 \%$ & 928 & $14.0 \%$ & 2,045 & $8.2 \%$ & 3,013 & $8.8 \%$ \\
\hline Miscellaneous & 28 & $1.2 \%$ & 804 & $12.2 \%$ & 295 & $1.2 \%$ & 1,127 & $3.3 \%$ \\
\hline Total & $\mathbf{2 , 4 3 0}$ & $\mathbf{1 0 0 \%}$ & $\mathbf{6 , 6 1 7}$ & $\mathbf{1 0 0 \%}$ & $\mathbf{2 5 , 0 4 1}$ & $\mathbf{1 0 0 \%}$ & $\mathbf{3 4 , 0 8 8}$ & $\mathbf{1 0 0 \%}$ \\
\hline
\end{tabular}

${ }^{1}$ TESS $=$ Transfusion Error Surveillance System

Approximately $98.6 \%(n=33,622)$ of all the errors were discovered before the actual blood transfusion, including all those related to sample collection $(n=13,680)$. The vast majority $(97.6 \%)$ of the 466 errors detected after the actual transfusion were from medium $(n=227)$ and large $(n=228)$ capacity hospitals. Hospitals transfusing less than 2,000 units annually accounted for only 11 cases (Table 2 ) which were errors related to inventory management $(n=2)$, request $(n=3)$ issuance $(n=2)$ and transfusion of prescribed products $(n=4)$.

Overall, the most frequently reported errors detected after transfusion were related to unit transfusions of which more than $69 \%(n=192)$ were from hospitals transfusing between 2,000 and 10,000 units annually (Table 2). Large capacity hospitals accounted for $29.5 \%(n=82)$ and the remaining $1.4 \%(n=4)$ originated from hospitals with smaller transfusion capacity (Table 2).

The least common of the transfusion errors detected after the actual transfusion were primarily errors related to product storage, distribution and the request for pick-up, which occurred only once or twice during the period between 2008 to 2011 (Table 2).

Table 2: Errors discovered AFTER $(n=466)$ blood transfusion by type of hospitals, TESS $2008-2011$

\begin{tabular}{|c|c|c|c|c|c|c|c|c|}
\hline \multirow[t]{2}{*}{$\begin{array}{c}\text { Type of Transfusion } \\
\text { error }\end{array}$} & \multicolumn{2}{|c|}{\begin{tabular}{|c|} 
Small \\
(less than 2,000 \\
transfusions per year) \\
\end{tabular}} & \multicolumn{2}{|c|}{$\begin{array}{c}\text { Medium } \\
(2,000 \text { to } 10,000 \\
\text { transfusions per year) }\end{array}$} & \multicolumn{2}{|c|}{$\begin{array}{c}\text { Large } \\
\text { (more than } 10,000 \\
\text { transfusions per year) }\end{array}$} & \multicolumn{2}{|c|}{ Overall } \\
\hline & Freq. & Rate & Freq. & Rate & Freq. & Rate & Freq. & Rate \\
\hline Distributor codes & 0 & - & 2 & 1: 117,432 & 0 & - & 2 & 1: 439,663 \\
\hline Product check-in & 0 & - & 0 & - & 3 & $1: 206,374$ & 3 & 1: 293,109 \\
\hline Unit storage & 0 & - & 0 & - & 1 & $1: 619,123$ & 1 & $1: 879,326$ \\
\hline Inventory management & 2 & 1: 12,670 & 3 & 1: 78,288 & 2 & 1: 309,562 & 7 & 1: 125,618 \\
\hline Product request & 3 & $1: 6,642$ & 5 & 1: 47,934 & 56 & 1: 11,103 & 64 & 1: 13,771 \\
\hline Request for pick-up & 0 & - & 0 & - & 1 & $1: 621,772$ & 1 & $1: 881,366$ \\
\hline Product selection & 0 & - & 3 & 1: 94,614 & 10 & $1: 65,484$ & 13 & 1: 73,786 \\
\hline Unit manipulation & 0 & - & 4 & 1: 70,961 & 2 & 1: 327,418 & 6 & 1: 159,870 \\
\hline Unit issue & 2 & 1: 9,264 & 11 & $1: 21,308$ & 30 & $1: 20,224$ & 43 & 1: 19,991 \\
\hline Unit transfusion & 4 & $1: 4,632$ & 192 & $1: 1,221$ & 82 & 1: 7,399 & 278 & $1: 3,092$ \\
\hline
\end{tabular}




\begin{tabular}{|l|c|c|c|c|c|c|c|c|}
\hline Sample handling & 0 & - & 0 & - & 7 & $1: 52,289$ & 7 & $1: 89,415$ \\
\hline Sample receipt & 0 & - & 4 & $1: 51,917$ & 18 & $1: 20,335$ & 22 & $1: 28,450$ \\
\hline Sample testing & 0 & - & 3 & $1: 112,172$ & 14 & $1: 58,353$ & 17 & $1: 70,731$ \\
\hline Miscellaneous & 0 & - & 0 & N/A & 2 & N/A & 2 & N/A \\
\hline Total & $\mathbf{1 1}$ & N/A & $\mathbf{2 2 7}$ & N/A & $\mathbf{2 2 8}$ & N/A & 466 & N/A \\
\hline
\end{tabular}

${ }^{1}$ TESS $=$ Transfusion Error Surveillance System

Since 2008, the total number of transfusion-related errors detected after the actual transfusion has changed significantly $(p<0.05)$. This number initially increased by almost $29 \%$ in 2009 and for the two following years, it decreased by $16 \%$ and $33 \%$ respectively (Table 3 ). Sixty-six (14.2\%) of these errors were deemed to be of high severity potential and of these, 16 resulted in adverse reactions which included eight cases of transfusionassociated circulatory overload, two cases of febrile non-hemolytic reaction, one case of mild transfusion reaction and five cases of unusual transfusion reactions (Table 4). None of these adverse reactions resulted in death.

Table 3: Errors discovered AFTER $(n=466)$ blood transfusion by surveillance year, TESS $2008-2011$

\begin{tabular}{|c|c|c|c|c|c|c|c|c|c|c|}
\hline \multirow{2}{*}{$\begin{array}{c}\text { Type of Transfusion } \\
\text { error }\end{array}$} & \multicolumn{2}{|r|}{2008} & \multicolumn{2}{|r|}{2009} & \multicolumn{2}{|r|}{2010} & \multicolumn{2}{|r|}{2011} & \multicolumn{2}{|c|}{ Overall } \\
\hline & Freq. & Rate & Freq. & Rate & Freq. & Rate & Freq. & Rate & Freq. & Rate \\
\hline Distributor codes & 0 & - & 0 & - & 2 & 1: 105,423 & 0 & - & 2 & 1: 439,663 \\
\hline Product check-in & 2 & 1: 115,521 & 0 & - & 0 & - & 1 & 1: 206,866 & 3 & 1: 293,109 \\
\hline Unit storage & 0 & - & 1 & 1: 230,572 & 0 & - & 0 & - & 1 & 1: 879,326 \\
\hline Inventory management & 2 & 1: 115,521 & 0 & - & 2 & 1: 105,423 & 3 & $1: 68,955$ & 7 & 1: 125,618 \\
\hline Product request & 18 & 1: 12,290 & 12 & 1: 19,068 & 16 & 1: 13,680 & 18 & 1: 11,802 & 64 & 1: 13,771 \\
\hline Request for pick-up & 1 & 1: 221,226 & 0 & - & 0 & - & 0 & - & 1 & 1: 881,366 \\
\hline Product selection & 1 & 1: 233,142 & 7 & $1: 34,177$ & 4 & 1: 59,301 & 1 & $1: 249,635$ & 13 & 1: 73,786 \\
\hline Unit manipulation & 1 & $1: 233,142$ & 0 & - & 4 & 1: 59,301 & 1 & 1: 249,635 & 6 & 1: 159,870 \\
\hline Unit issue & 10 & $1: 21,485$ & 14 & 1: 15,937 & 11 & 1: 19,621 & 8 & $1: 25,728$ & 43 & 1: 19,991 \\
\hline Unit transfusion & 63 & $1: 3,410$ & 95 & $1: 2,349$ & 78 & $1: 2,767$ & 42 & 1: 4,901 & 278 & 1: 3,092 \\
\hline Sample handling & 1 & 1: 166,703 & 2 & 1: 84,971 & 3 & 1: 49,982 & 1 & 1: 139,317 & 7 & $1: 89,415$ \\
\hline Sample receipt & 4 & 1: 41,676 & 5 & $1: 33,988$ & 7 & $1: 21,421$ & 6 & $1: 23,220$ & 22 & $1: 28,450$ \\
\hline Sample testing & 5 & $1: 33,341$ & 3 & 1: 56,647 & 5 & 1: 29,989 & 4 & 1: 34,829 & 17 & 1: 70,731 \\
\hline Miscellaneous & 0 & N/A & 0 & N/A & 0 & N/A & 2 & N/A & 2 & N/A \\
\hline Total & 108 & N/A & 139 & N/A & 132 & N/A & 87 & N/A & 466 & $N / A$ \\
\hline
\end{tabular}

${ }^{1}$ TESS $=$ Transfusion Error Surveillance System

Table 4: Specific errors that resulted in adverse reactions, TESS $^{1} 2008-2011$

\begin{tabular}{|l|c|c|c|c|c|}
\hline \multicolumn{1}{|c|}{ Transfusion Error } & $\begin{array}{c}\text { Transfusion-associated } \\
\text { circulatory overload }\end{array}$ & $\begin{array}{c}\text { Febrile non- } \\
\text { hemolytic }\end{array}$ & $\begin{array}{c}\text { Mild transfusion } \\
\text { reaction }\end{array}$ & $\begin{array}{c}\text { Transfusion reaction } \\
\text { of unspecified nature }\end{array}$ & Total \\
\hline Incorrect/No product order & 1 & 0 & 0 & 0 & 1 \\
\hline Inappropriate product order & 5 & 1 & 0 & 2 & 8 \\
\hline
\end{tabular}




\begin{tabular}{|l|c|c|c|c|c|}
\hline Incorrect/No historical review & 0 & 0 & 0 & 1 & 1 \\
\hline $\begin{array}{l}\text { Wrong product issued to a } \\
\text { patient }\end{array}$ & 0 & 0 & 0 & 2 & 2 \\
\hline $\begin{array}{l}\text { Transfusion of product with } \\
\text { incompatible fluid }\end{array}$ & 0 & 1 & 0 & 0 & 1 \\
\hline $\begin{array}{l}\text { Guidelines for infusion time } \\
\text { not followed }\end{array}$ & 2 & 0 & 1 & 0 & 3 \\
\hline Total & $\mathbf{8}$ & $\mathbf{2}$ & $\mathbf{1}$ & $\mathbf{5}$ & $\mathbf{1 6}$ \\
\hline
\end{tabular}

${ }^{1}$ TESS $=$ Transfusion Error Surveillance System

\section{Conclusion}

The TESS has demonstrated that participating hospitals have a very robust transfusion safety system in place as evidenced by data collected over a four-year period which showed that only a tiny proportion of transfusionrelated errors go undetected prior to the actual blood transfusion. Moreover, the frequency of these errors has been decreasing since 2010. Continued monitoring of transfusion errors will support transfusion and patient safety and will also allow for the identification of points in the transfusion chain where targeted quality improvement initiatives would enable continuous decrease of the frequency of transfusion errors, particularly those escaping detection before the actual transfusion.

\section{Acknowledgements}

The development of the Transfusion Error Surveillance System (TESS) would not have been possible without the collaborative support and continued commitment of many transfusion safety officers, medical laboratory technologists and other healthcare professionals in hospitals and blood transfusion services. Their dedication to reducing errors and increasing patient safety has led to the collection and analysis of the 2008 - 2011 TESS data.

\section{Conflict of interest}

None

\section{Funding}

This work was supported by the Public Health Agency of Canada.

\section{Reference}

(1) Public Health Agency of Canada. Transfusion Error Surveillance System (TESS) - 2008-2011 Report. Ottawa: Centre for Communicable Diseases and Infection Control, PHAC; 2014.

http://www.phac-aspc.gc.ca/hcai-iamss/tess-sset/results-resultats-2008-2011-eng.php 